\title{
Ovarian cyst haemorrhage as a complication of acute myelomonocytic leukaemia induction therapy
}

\author{
Authors: Amy L Irwin, ${ }^{A}$ Katherine Smith ${ }^{B}$ and Nigel Sargant ${ }^{C}$
}

\section{Background}

Here we present a case of acute ovarian cyst haemorrhage in a young female during induction therapy for acute myelomonocytic leukaemia (AMML).

\section{Case presentation}

A patient undergoing chemotherapy on the AML19 trial for AMML developed severe abdominal pain and haemodynamic compromise during cycle 2 of fludarabine, cytarabine and idarubicin. The patient was found to have a large ruptured haemorrhagic ovarian cyst on computed tomography. She was managed conservatively due to relative haematological contraindications to surgery and haemodynamic stability following transfer to the high dependency unit. The patient had recently discontinued anticoagulation for pulmonary emboli due to thrombocytopenia.

\section{Conclusions}

This highlights the importance of recognising coexistent pathology in patients undergoing high intensity chemotherapy.

KEYWORDS: AMML, chemotherapy, ovarian cyst haemorrhage, thrombocytopenia, anticoagulation

\section{Case presentation}

A previously fit 41-year-old woman with no previous history of bleeding problems presented with a high white cell count of $304 \times 10^{9} / \mathrm{L}$ and normal coagulation screen. A diagnosis of acute myelomonocytic leukaemia (AMML) was made. Subsequent investigations revealed the patient had normal cytogenetics by G-banding with an associated FLT3-ITD mutation plus NUP98NSD1 (defined in trial). She consented to go into AML19 and was randomised to receive fludarabine, cytarabine and idarubicin (Fla-Ida) and gemtuzumab ozogamicin on her first cycle.

At diagnosis, her current medication was the oral contraceptive pill (desogestrel). She was found to have bilateral

Authors: ${ }^{\text {A }}$ core medical trainee, North Hampshire and Basingstoke Hospital, Basingstoke, UK; ${ }^{B}$ consultant haematologist, North Hampshire and Basingstoke Hospital, Basingstoke, UK; ${ }^{C}$ consultant haematologist and blood transfusion lead, North Hampshire and Basingstoke Hospital, Basingstoke, UK pulmonary emboli diagnosed during her initial work-up and anticoagulation was commenced. This continued while her platelet counts remained satisfactory. Due to the thrombosis risk and the perceived increased incidence of vaso-occlusive disease with oral contraceptive pill and gemtuzumab ozogamicin, the desogestrel was stopped.

She had several complications prior to and throughout her induction chemotherapy. These included chest sepsis, pulmonary emboli, menorrhagia, intractable nausea and electrolyte derangement requiring high dependency unit support. She achieved a morphological remission post cycle ${ }^{1}$ Fla-Ida and gemtuzumab ozogamicin. She remained minimal residual disease positive with ongoing detection of NUP98-NSD1.

She was readmitted for cycle 2 Fla-Ida and disclosed that she had not had any menses for the previous 6 weeks. A pregnancy test was performed which was negative and her chemotherapy commenced. Her anticoagulation (subcutaneous enoxaparin) was continued while her platelets remained above $50 \times 10^{9} / \mathrm{L}$. Her enoxaparin was then subsequently held.

Five days into her treatment with Fla-Ida she developed severe, rapidly worsening, intractable left lower quadrant and loin pain which radiated into her vagina, she had difficulty with micturition and she became haemodynamically compromised.

An urgent computed tomography (CT) was requested identifying a large haematoma / fluid complex collection centred at the left adnexa representing a large ruptured ovarian cyst (Fig 1).

CT angiography identified no area amenable to embolisation. A decision was made to manage her conservatively given the patient was pancytopaenic and haemodynamically stabilised. She was supported with analgesia, antibiotics, tranexemic acid and blood products to maintain haemoglobin of $70 \mathrm{~g} / \mathrm{L}$ and platelets of $>50 \times 10^{9} / \mathrm{L}$

Ruptured ovarian cysts are a relatively common phenomenon in menstruating women, but rarely do they become haemorrhagic or cause such disabling or sudden serious symptoms. It is a complication that physicians should be aware of when managing women of childbearing age with chemotherapy and anticoagulation.

Our case demonstrates that a usually benign and minor phenomenon can have serious and potentially life-threatening consequences. It highlights the importance of recognising and responding to coexistent pathology in younger female patients requiring high intensity chemotherapy treatment for AMML. 
Fig 1. Computed tomography identifying a large haematoma / fluid complex collection centred at the left adnexa. a) Image from front. b) Image from side.
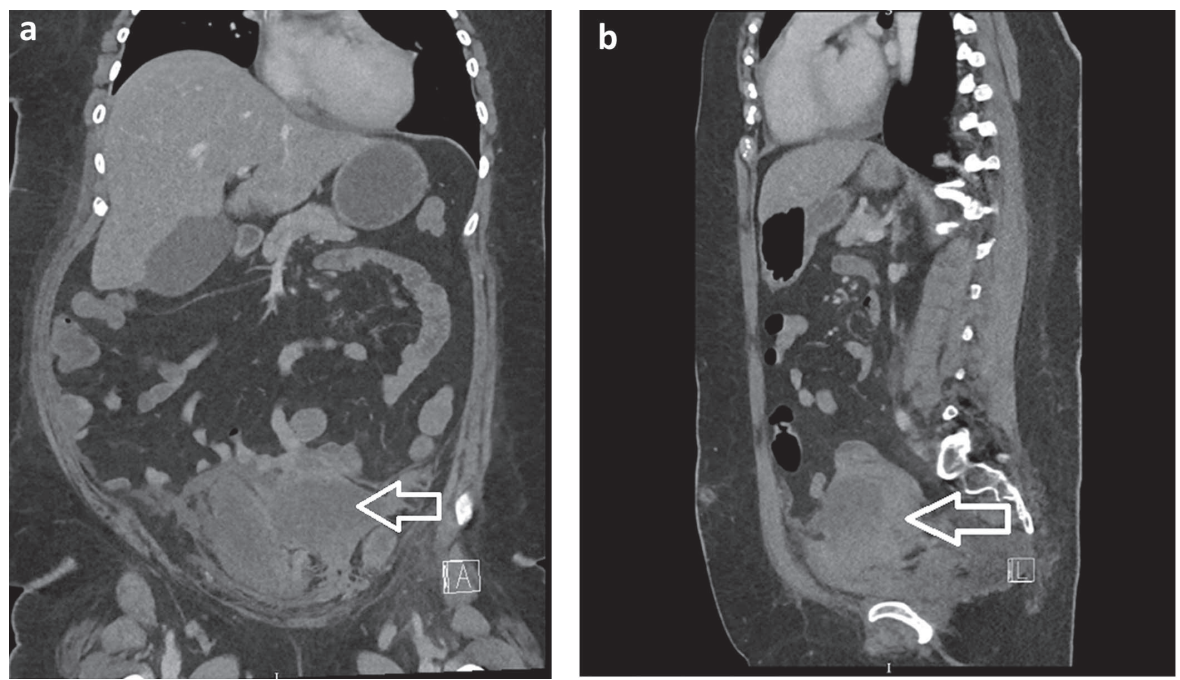

Ovarian cysts are known to be common in women of child bearing age with $4 \%$ of women being admitted to hospital for an ovarian cyst by the age of 65 years. ${ }^{1}$

Complications in the general population of haemoperitoneum and haemodynamic compromise are rare, but risk factors for cyst haemorrhage reported in normal patients include anticoagulation therapy, congenital and acquired thrombophilia and early pregnancy. ${ }^{1}$ -

\section{Reference}

1 Bottomley C, Bourne T. Diagnosis and management of ovarian cyst accidents. Best Pract Res Clin Obstet Gynaecol. 2009;23:711-24.

Address for correspondence: Dr Amy Louise Irwin, North Hampshire and Basingstoke Hospital, Haematology department, Aldermaston Road, Basingstoke RG24 9NA, UK. Email: amy.irwin@hhft.nhs.uk

\section{Royal College} of Physicians

\section{Apply for the 2020 Turner-Warwick lecturer scheme}

Celebrating the life and achievements of the Royal College of Physicians' (RCP's) first female president, Dame Margaret Turner-Warwick, applications for the 14 lectureships are now open.

A lecturer will be selected from the trainees applying in each region to present to peers and senior colleagues at the regional Update in medicine (or similar meeting).

For full details visit: rcplondon.ac.uk/educationpractice/funding-awards/turner-warwick-lectures

\section{Lecturers will be given:}

$>$ the opportunity to present at a regional RCP meeting

$>$ a plaque presented by Professor Andrew Goddard, president of the RCP

> entry in the Turner-Warwick yearbook

$>$ an invitation to the Harveian Oration and dinner.

The overall winner will be invited to present at the RCP's annual conference, Medicine 2021.

Enquiries: UKregions@rcplondon.ac.uk

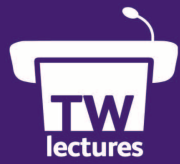

